\title{
Verzeichnis der Bearbeiter der 10. Auflage
}

Erwin Abele, Rechtsanwalt in München

Dr. Christian Armbrüster, Professor an der Freien Universität Berlin

Dr. Frank Baumann, LL.M., Rechtanwalt und Fachanwalt für Versicherungsrecht in Hamm

Dr. Horst Baumann $(\dagger)$, emeritierter Professor an der Technischen Universität Berlin

Dr. Roland Michael Beckmann, Professor an der Universität des Saarlandes, Saarbrücken

Dr. Oliver Brand, LL.M. (Cambridge), Professor an der Universität Mannheim

Dr. Alexander Bruns, LL.M. (Duke Univ.), Professor an der Albert-Ludwigs-Universität Freiburg

Dr. Christoph Brömmelmeyer, Professor an der Europa-Universität Viadrina Frankfurt (Oder)

Dr. Jan Dreyer, Rechtsanwalt und Fachanwalt für Versicherungsrecht in Hamburg

Charlotte Echarti, Bereichsleiterin Run Off Solutions, E+S Rückversicherung AG, Hannover

Christiane Eifler, LL.M., Rechtsanwältin in Nürnberg

Anne Fischer, LL.M., Rechtsanwältin in Düsseldorf

Dr. Thomas Gädtke, Rechtsanwalt in München und Lehrbeauftragter an der LudwigMaximilians Universität München

Dr. Jens Gal, Maître en droit (Lyon 2), Privatdozent und Lehrstuhlvertreter an der GoetheUniversität, Frankfurt am Main

Dr. Maximilian Guth, LL.M. (Southampton), Rechtsanwalt in Hamburg, Solicitor of England \& Wales

Dr. Olaf Hartenstein, D.E.A. (Sorbonne), LL.M. (Assas), Rechtsanwalt in Hamburg

Dr. Dr. h.c. Helmut Heiss, Professor an der Universität Zürich und Rechtsanwalt in Zürich

Dr. Jörg Henzler, Rechtsanwalt in Stuttgart

Dr. Harald Herrmann, emeritierter Professor an der Friedrich-Alexander-Universität ErlangenNürnberg

Dr. Detlef A. Huber, Rechtsanwalt in Freiburg i.Br.

Jens Jaeger, Rechtsanwalt in Hamburg, Lehrbeauftragter an der Universität Hamburg

Dr. Ralf Johannsen ( $\dagger$ ), Rechtsanwalt in Hamburg

Dr. Katharina Johannsen $(\dagger)$, Vorsitzende Richterin am Hanseatischen OLG a. D., Hamburg

Dr. Rocco Jula, Rechtsanwalt und Fachanwalt für Versicherungsrecht in Berlin

Dr. Kai-Oliver Knops, Professor an der Universität Hamburg

Dr. Robert Koch, LL.M. (McGill), Professor an der Universität Hamburg

Dr. Hubertus W. Labes, Rechtsanwalt in Hamburg, Lehrbeauftragter an der Universität Hamburg

Dr. Annemarie Matusche-Beckmann, Professorin an der Universität des Saarlandes, Saarbrücken

Dr. Ernst Niederleithinger, Ministerialdirektor beim Bundesministerium der Justiz a. D., Honorarprofessor der Ruhr-Universität Bochum

Dr. Stefan Perner, Professor und Vorstand am Institut für Zivil- und Zivilverfahrensrecht an der Wirtschaftsuniversität Wien

Jürgen Raab, Rechtsanwalt in Hamburg

Dr. Jens-Berghe Riemer, Rechtsanwalt und Fachanwalt für Versicherungsrecht sowie Transport- und Speditionsrecht in Nürnberg

Dr. Claus von Rintelen, Rechtsanwalt und Fachanwalt für Versicherungsrecht in Hamburg

Dr. Christian Rolfs, Professor an der Universität zu Köln

Dr. Christian Schneider, Rechtsanwalt und Fachanwalt für Versicherungsrecht in Köln

Dr. Winfried Schnepp, Rechtsanwalt und Fachanwalt für Versicherungsrecht in Köln

Arno Schubach, Rechtsanwalt und Fachanwalt für Versicherungsrecht in Frankfurt am Main

Dr. Dieter Schwampe, Professor an der Universität Hamburg, Rechtsanwalt in Hamburg

Dr. Hans-Peter Schwintowski, Professor an der Humboldt-Universität zu Berlin

Dr. Torsten Sommer, Richter am Landgericht Hamburg

Dr. Ansgar Staudinger, Professor an der Universität Bielefeld 
Dr. Wolfgang Voit, Professor an der Philipps-Universität Marburg

Dr. Conrad Waldkirch, akademischer Rat a.Z. an der Universität Mannheim

Dr. Gerrit Winter, emeritierter Professor an der Universität Hamburg 